\title{
3-Hydroxycoumarin as a New Matrix for Matrix-Assisted Laser Desorption/Ionization Time-of-Flight Mass Spectrometry of DNA
}

\author{
Zhenying Zhang, ${ }^{*}$ Lihua Zhou, ${ }^{*}$ Shankai Zhao, and Huimin Deng \\ Instrumental Analysis and Research Center, Sun Yat-Sen University, Guangdong, People's Republic of China
}

\author{
Qinying Deng \\ School of Chemistry and Chemical Engineering, Sun Yat-Sen University, Guangdong, People's Republic \\ of China
}

\begin{abstract}
3-Hydroxycoumarin (3-HC) was designed, synthesized, and tested as a matrix for matrixassisted laser desorption/ionization time-of-flight mass spectrometry (MALDI-TOF MS) analyses of a variety of synthetic oligodeoxynucleotides ranging long from three to 70 bases. Using the matrix solution of 3-HC dissolved in a mixed solvent of acetone and diammonium hydrogen citrate, DNA segments over the mass range 800 Da to 6900 Da were isotopically resolved with high signal-to-noise $(\mathrm{S} / \mathrm{N})$ ratio. The individual isotopic molecular ion peaks of a group of 23-mer mixed-base oligomers differing by one or two bases with mass differences of 9 or $7 \mathrm{Da}$ were recorded. Larger oligodeoxynucleotide segments of 34-mer, 50-mer, and 70-mer have also been analyzed effectively. Less than 250 attomol of a 10-mer DNA segment was clearly detected without any fragmentation. The new matrix can be used for the analysis of DNA segments in both positive- and negative-ion modes, and the quality of all negative-ion mode spectra are as good as that obtained in positive-ion mode shown in this paper. Compared with conventional matrices of 3-hydroxypicolinic acid (3-HPA) and 6-aza-2thiothymine (ATT), 3-HC had noticeable improvement in resolution, S/N ratio, spot-to-spot-, and sample-to-sample reproducibility for analyzed DNA segments. (J Am Soc Mass Spectrom 2006, 17, 1665-1668) (C) 2006 American Society for Mass Spectrometry
\end{abstract}

$\mathrm{M}$ ALDI-TOF MS is a useful tool for the analysis of synthetic oligonucleotides [1], DNA sequencing [2], single-nucleotide polymorphism (SNP) genotyping [3, 4], and microsatellite analysis [5]. At this time, despite extensive research, matrices for practical usage in DNA detection have been limited to 3-hydroxypicolinic acid (3-HPA) [6], picolinic acid (PA) [7], 6-aza-2-thiothymine (ATT) [8], and 2,4,6-trihydroxyacetophenone (2,4,6-THP) [9].

The introduction of 3-HPA as a matrix, initially used to analyze a 67-mer DNA [6], has greatly increased MALDI MS ability to analyze DNA. Chen and coworkers [7] discovered PA and applied it to analyze mixedbase oligonucleotides up to 190 bases and RNA $^{\text {phe }}$ with 76 bases. ATT is appropriate to achieve well-resolved spectra and can detect double-stranded DNA as a "cool" matrix [8, 10]. 2,4,6-THP or a mixture of $2,4,6-$ THP, 2,3,4-trihydroxyacetophenone, and ammonium

Published online August 14, 2006

Address reprint requests to Dr. H. Deng, Instrumental Analysis and Research Center, Sun Yat-Sen University, Guangzhou 510275, Guangdong, P.R. China. E-mail: denghm@mail.sysu.edu.cn

* Also at the School of Chemistry and Chemical Engineering, Sun Yat-Sen University. citrate were demonstrated to have good tolerance to metal salts $[9,11]$. Quinaldic acid (QA) was found to be a useful matrix for DNA or RNA by Song [12]. Some co-component matrices and sample preparation methods, such as a mixture of 3-HPA and pyrazinecarboxylic acid [13] and fucose as additive to DNA sample solution [14], or thin-layer sample preparation with ATT as the matrix [8], were applied in DNA analysis by MALDI MS to produce more homogeneous sample spots, better resolution, and excellent reproducibility.

In the present work, we demonstrate a new reagent, 3-HC, to be an excellent matrix for MALDITOF MS analysis of pure and mixed single-stranded oligonucleotides.

\section{Experimental}

Design, Synthesis, and Characterization of 3-Hydroxycoumarin

3-HC (the molecular structure is shown in Scheme 1) was designed based on the structures of the most successful matrices such as sinapinic acid (SA), $\alpha$-cyano-4hydroxycinnamic acid (HCCA), 2,5-dihydroxybenzoic 
<smiles>O=c1oc2ccccc2cc1O</smiles>

$3-\mathrm{HC}$

Scheme 1. Molecular structure of 3-HC.

acid (DHB), 3-HPA, and 2,4,6-THP. Similar to SA and HCCA, 3-HC contains the conjugated system composed of a phenyl ring and $\alpha, \beta$-unsaturated ketone group, which is anticipated to have sufficient absorption for $337 \mathrm{~nm}$ laser, and simultaneously, 3-HC has the hydroxyl and carbonyl groups, regarded as important functional groups for a good matrix of DNA [15], the two adjacent function groups being similar to the orthopositioned structure between hydroxyl group and carbonyl group in DHB, 3-HPA, and 2,4,6-THP molecules to a certain extent.

3-HC was synthesized following the literature [16], and structure identification was confirmed by IR and NMR. Its most intense absorption band is at $308 \mathrm{~nm}$ with extinction coefficient $(\varepsilon)$ of $1.075 \times 10^{4} \mathrm{~L} \mathrm{~mol}^{-1} \mathrm{~cm}^{-1}$.

\section{Mass Spectrometric Analyses}

MALDI-TOF MS analyses were performed on a REFLEX III MALDI-TOF mass spectrometer (Bruker, Bremen, Germany) with a nitrogen laser at a wavelength of $337 \mathrm{~nm}$. The acceleration and detection potentials were set at 20 and $1.55 \mathrm{kV}$, respectively. The laser power was adjusted during experiments; 40 single laser spots were averaged for each spectrum. The resolution calculations were made by full width half maximum (FWHM). Signal-to-noise (S/N) was calculated by the spectrum process software XMASS 5.1 supplied by Bruker. The narrowest monoisotopic peak was chosen to calculate resolution and $\mathrm{S} / \mathrm{N}$ for well-resolved isotopic signals. The resolution of the unresolved isotopic peaks was calculated based on the unresolved isotope cluster. Larger oligodeoxynucleotide segments of 34mer, $\mathrm{d}(\mathrm{T})_{50}$, and 70-mer, were analyzed in linear mode since the detection power of the instrument decreases when analyzing higher mass samples, and other DNA segments were analyzed in reflectron mode.

\section{MALDI Sample Preparation}

Three 23-mer oligonucleotides were purchased from Biosia Ltd. (Shanghai, China), and the other oligonucleotides were purchased from The Institute of Microbiology, Chinese of Academy of Sciences (Beijing, China). All DNA samples were of HPLC purity and used as received. After investigating the 3-HC matrix solutions prepared at different concentrations by a variety of solvents, we found $0.15 \mathrm{M}$ 3-HC prepared with the mixed solvents of acetone and $0.1 \mathrm{M}$ diammonium hydrogen citrate (DHC, which is commonly added to matrix solution or synthesized oligonucleotides to reduce metal adducts formation) ( $\mathrm{vol} / \mathrm{vol}=1: 1)$ resulted in high quality spectra. 3-HC was recrystallized from deionized water before use. For comparison, 3-HPA and ATT (Aldrich, Milwaukee, WI) were also used and each was dissolved in mixed solvents of $50 \%$ acetonitrile and 50\% 0.1 M DHC aqueous solution to a saturated solution. A $0.5 \mu \mathrm{L}$ mixture of equal volumes of $10^{-5} \mathrm{M}$ DNA in deionized water and of the matrix solution was deposited on a stainless target and dried at room-temperature for MALDI TOF MS analysis.

\section{Results and Discussion}

\section{MALDI-TOF MS Analyses of Pure Single- Stranded DNA Segments}

The positive-ion and reflectron mode mass spectra of five 10-mer oligonucleotides including $\mathrm{d}(\mathrm{T})_{10}, \mathrm{~d}(\mathrm{C})_{10}$, $\mathrm{d}(\mathrm{A})_{10}, \mathrm{~d}(\mathrm{G})_{10}$, and a 10-mer (5'ATC GAT CGA T 3') were acquired with $3-\mathrm{HC}$ matrix. Figure 1 presents the spectrum of a 10-mer: a representative spectrum of the five DNA segments with 10 bases long.

Postsource decay contributes to peak broadening and results in difficulties for MALDI TOF MS analysis of DNA segments with molecular weights greater than $5000 \mathrm{Da}$ in reflectron mode. Metal adducts of DNA segments usually result in illegible molecular ion peaks and reduce the spectrum resolution. In the present work, for DNA segments with mass up to $\sim 7000 \mathrm{Da}$, typically, a resolution higher than 10,000 and $\mathrm{S} / \mathrm{N}$ value higher than 800 could be achieved with 3-HC as matrix in reflectron mode. For comparisons, 3-HC, ATT, and 3-HPA were used to analyze $\mathrm{pd}(\mathrm{T})_{18}, \mathrm{pd}(20$-mer)(5'TTA CTC TGT TAA TGT CTT TG $\left.3^{\prime}\right)$, and 23-mer (C) (5'TCC ACC ATT AGC ACC CAA AGC TA 3') in reflectron mode under identical experimental condition. Figure 2 compares the resulting mass spectra of 23-mer (C). As is shown in Figure 2a and b, isotopic molecular ions could not be acquired by using ATT or 3-HPA, and the best quality spectrum with isotopically resolved peaks was

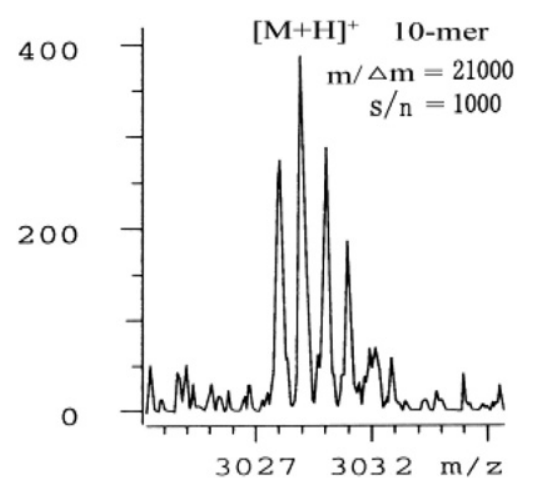

Figure 1. Positive-ion MALDI-TOF mass spectrum of 10-mer using 3-HC as matrix in reflectron mode 


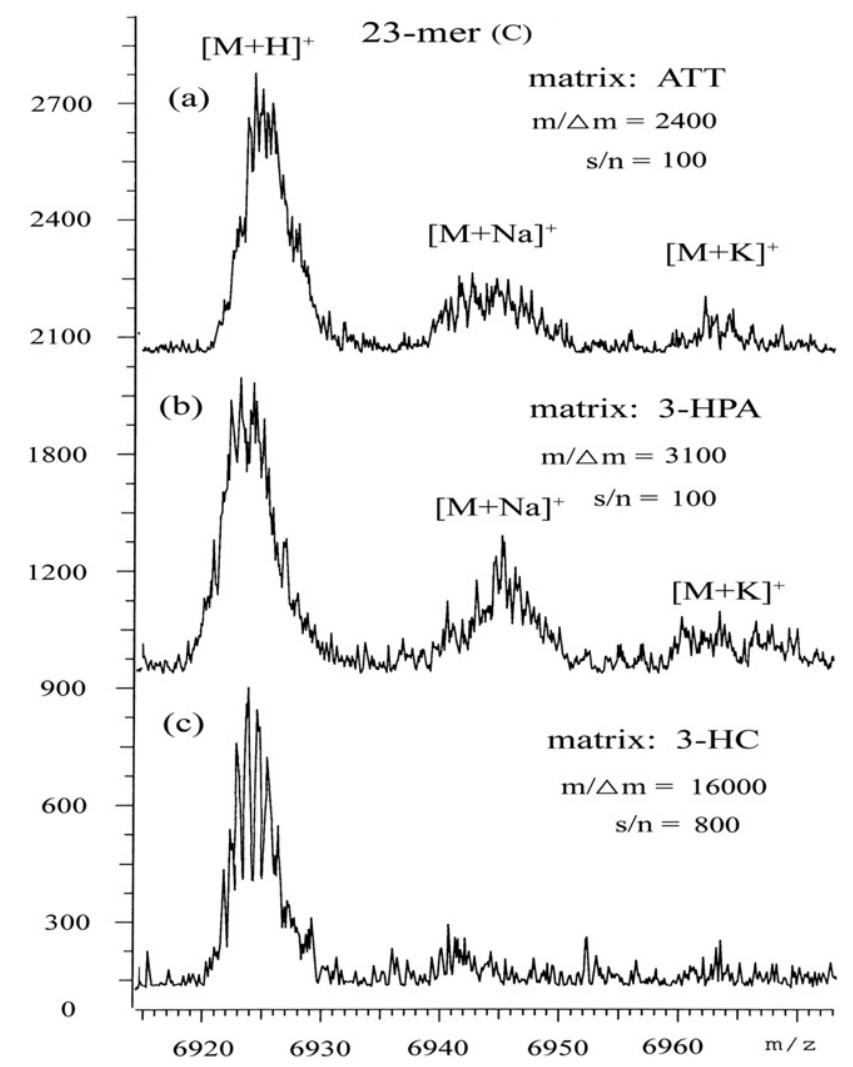

Figure 2. Positive-ion MALDI-TOF mass spectra of 23-mer (C) in reflectron mode. Matrix: (a) ATT, (b) 3-HPA, and (c) 3-HC .

obtained using 3-HC as matrix. Furthermore, in Figure $2 \mathrm{a}$ and $\mathrm{b}$, the adduct ions of $[\mathrm{M}+\mathrm{Na}]^{+}$and $[\mathrm{M}+\mathrm{K}]^{+}$ were observed, while in Figure $2 \mathrm{c}$ obtained with $3-\mathrm{HC}$ as matrix, similar adducts were not observed. The results suggest that $3-\mathrm{HC}$ has the effect to reduce metal adduct ions formed.

Larger DNA segments of 34-mer (5'GGG TTC GAA TGA GCA GTA TTT CTG ATC AGG ACC C3'), d (T) 50 , and 70-mer (5'GAT CAA GCT TAT GTA CCC ATA CGA CGT CCC AGA CTA CGC TGT GAT GGG AAT GCT CCT GTC ATT CTT ACC T 3', 21 426.0 Da) were effectively ionized by 3-HC. The linear spectra (not shown) of $d(T)_{50}$ with $3-\mathrm{HC}$ and 3-HPA as matrixes, respectively, were obtained. To get the molecular ion signals of $d(T)_{50}$ with $3-\mathrm{HC}$ as matrix, about $15 \%$ lower laser power was needed in contrast to using 3-HPA, resulting in less fragments produced and the comparatively low intensity of fragment ion peak.

In addition to strong molecular ion peak and doubly charged ion, dimer, and even trimer of 70-mer up to 64,000 Da were recorded in positive-ion mode. Thus, single-stranded-DNA segments considerably larger than 70 bases are believed to be readily analyzed using 3-HC as matrix.

\section{MALDI-TOF MS Analyses of the DNA Mixtures}

The analysis of DNA mixtures is more difficult than that of single-component DNA segments by MALDI MS [17]. Thus, an appropriate matrix for the analysis of DNA mixture must satisfy two requirements. First, multiply charged fragments and adduct ions must appear small in magnitude and frequency. Second, the mass spectra must have enough resolution to distinguish individual peaks. The positive-ion mass spectrum of a mixture of $\mathrm{d}(\mathrm{A})_{3}-\mathrm{d}(\mathrm{A})_{10}$ is shown in Figure 3. Each molecular ion peak of $d(A)_{3}-d(A)_{10}$ is clearly discerned without fragment ions, cluster ions and adduct ions.

MALDI MS is being used for disease diagnoses by oligonucleotide analysis and characterization of single nucleotides polymorphisms (SNPs). Resolution to one base is required in these applications. A three-component mixture of 23-mer (A) (5'TCC ACC ATT AAC ACC CAA AGC TA 3', 6 905.6 Da), 23-mer (B) (5'TCC ACC ATT TGC ACC CAA AGC TA 3', $6912.6 \mathrm{Da})$, and

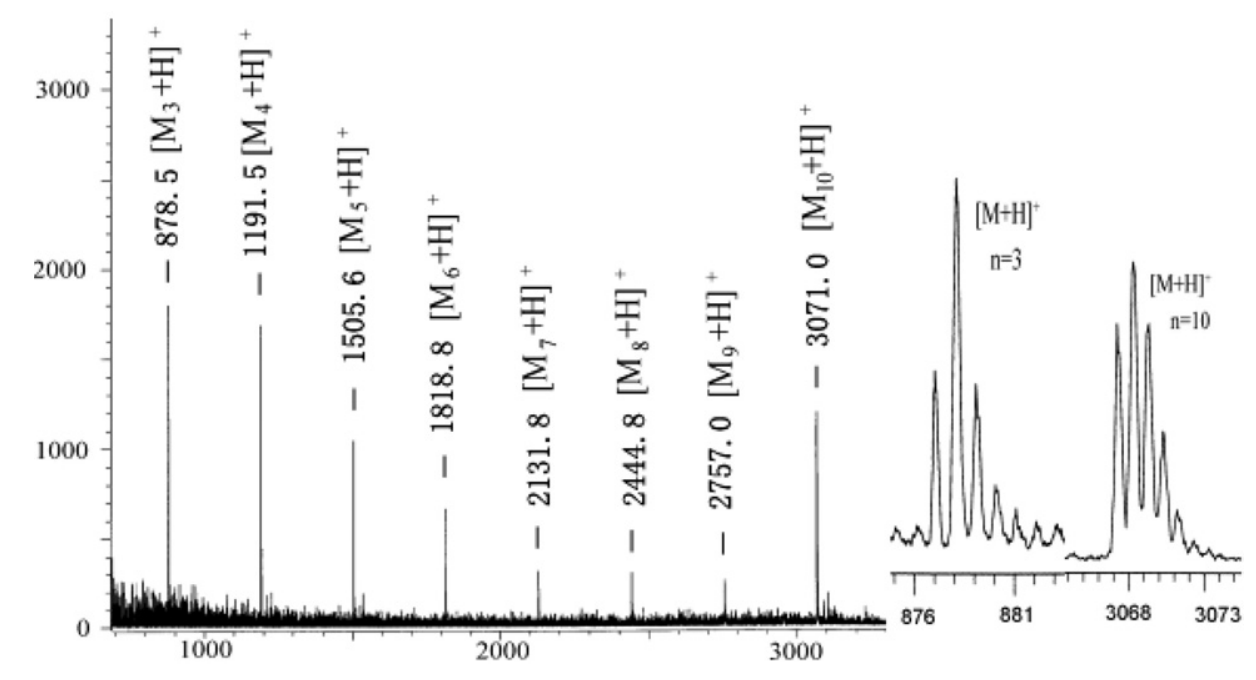

Figure 3. Positive-ion MALDI-TOF mass spectra of $d(A)_{3}-d(A)_{10}$ mixture with 3-HC as matrix in reflectron mode. The enlarged mass spectra show the mass peaks of $d(A)_{3}$ and $d(A)_{10}$, respectively. 


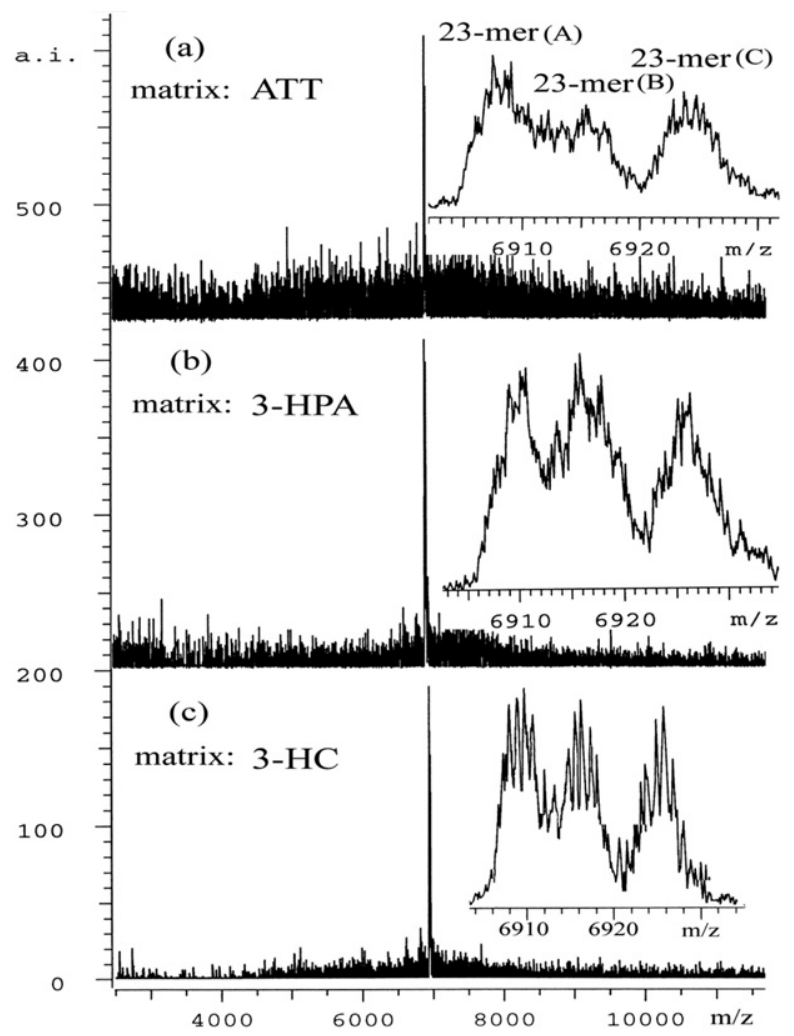

Figure 4. Positive-ion MALDI-TOF mass spectra of the threecomponent mixture of 23-mer (A), 23-mer (B), and 23-mer (C) in reflectron mode. Matrix: (a) ATT, (b) 3-HPA, and (c) 3-HC.

23-mer (C) (5'TCC ACC ATT AGC ACC CAA AGC TA $3^{\prime}, 6921.6 \mathrm{Da}$ ) mixture with the mass difference of $7 \mathrm{Da}$ [AA in 23-mer (A) versus TG in 23-mer (B)] and 9 Da [T in 23-mer (B) versus $A$ in 23-mer (C)] were analyzed with $3-\mathrm{HC}$ as matrix, and the results were compared with those obtained from ATT and 3-HPA as matrices under the same experimental condition. Figure 4 shows the mass spectra of the mixture of 23-mer (A), 23-mer (B), and 23-mer (C). It is obvious that the traces in Figure 4c obtained by 3-HC have relatively low noise, and not only is each component peak completely separated, but also their individual isotopic peaks are well resolved. The traces in Figure $4 \mathrm{~b}$ with 3 -HPA as matrix show that each component peak can be separated from each other, but the isotopic peaks cannot be resolved. The spectra of Figure 4a with ATT as matrix show each component peak cannot be separated sufficiently and the baseline noise is obviously higher than that observed in Figure 4c.

\section{Sensitivity}

The positive-ion mass spectra of a $2.5 \times 10^{-16} \mathrm{~mol}$ of 10-mer with 3-HC and 3-HPA as matrices in reflectron mode summed by 200 laser shots were recorded, respectively. The sensitivity experiment showed that using the same laser power, 3-HC resulted in a molecular ion peak with $\mathrm{S} / \mathrm{N}$ of 40 and without any sign of fragmentation, while no molecular ion was observed with 3-HPA. Increasing $20 \%$ of the laser power allowed the observation of the molecular ion with 3-HPA, but at the same time fragment ion peaks assigned to $\mathrm{pd}(\mathrm{TAG})$ $+\mathrm{Na}$ pd $(\mathrm{TAG})+3-\mathrm{HPA}+\mathrm{Na}$, pd(TAGC) + 3-HPA-80 $+\mathrm{H}^{\prime} \mathrm{pd}(\mathrm{TAGC})+3-\mathrm{HPA}+\mathrm{K}$, and pd(TAGCT) + 3-HPA- $80+\mathrm{H}$ presented.

\section{Acknowledgments}

This work was supported by the Guangdong Province Natural Sciences Foundation Council of China (grant no.990209). The authors thank senior engineer Jinan Chen and the College of Life Science of Xiamen University for supplying them with MALDITOF MS instrument to perform the experiments of sensitivity and negative-ion analyses. The authors are grateful to Professor Peixuan Guo and Professor Weiguo Andy Tao of Purdue University for their kind help.

\section{References}

1. Guo, B. C. Mass Spectrometry in DNA Analysis. Anal. Chem. 1999, 71, 333R-337R.

2. Bentzley, C. M.; Johnston, M. V.; Larsen, B. S.; Gutteridge, S. Oligonucleotide Sequence and Composition Determined by Matrix-Assisted Laser Desorption/Ionization Time-of-Flight Mass Spectrometry. Anal. Chem. 1996, 68, 2141-2146.

3. Sauer, S.; Gut, I. G. Genotyping Single-Nucleotide Polymorphisms by Matrix-Assisted Laser Desorption/Ionization Time-of-Flight Mass Spectrometry. Int. J. Chromatogr. B 2002, 782, 73-87.

4. Tang, K.; Opalsky, D.; Abel, K.; van den Boom, D.; Yip, P.; Mistro, G. D.; Braun, A.; Cantor, C. R. Single Nucleotide Polymorphism Analyses by MALDI-TOF MS. J. Mass Spectrom. 2003, 226, 37-54.

5. Taranenko, N. I.; Golovlev, V. V.; Allman, S. L.; Taranenko, N. V.; Chen, C. H.; Hong, J.; Chang, L. Y. Matrix-Assisted Laser Desorption/ Ionization for Short Tandem Repeat Loci. Rapid Commun. Mass Spectrom. 1998, 12, 413-418.

6. Wu, K. J.; Steding, A.; Becker, H. Matrix-Assisted Laser Desorption Time-of Flight Mass Spectrometry of Oligonucleotides Using 3-Hydroxypicolinic Acid as an Ultraviolet-Sensitive Matrix. Rapid Commun. Mass Spectrom. 1993, 7, 142-146

7. Tang, K.; Taranenko, N. I.; Allman, S. L.; Chen, C. H.; Chang, L. Y.; Jacobson, K. B. Picolinic Acid as a Matrix for Laser Mass Spectrometry of Nucleic Acids and Proteins. Rapid Commun. Mass Spectrom. 1994, 8, 673-677.

8. Garcia, B. A.; Heaney, P. J.; Tang, K. Improvement of the MALDI-TOF Analysis of DNA with Thin-Layer Matrix Preparation. Anal. Chem. 2002, 74, 2083-2091.

9. Lavanant, H.; Lange, C. Sodium-Tolerant Matrix for Matrix-Assisted Laser Desorption/Ionization Mass Spectrometry and Postsource Decay of Oligonucleotides. Rapid Commun. Mass Spectrom. 2002, 16, 1928-1933.

10. Distler, A. M.; Allison, J. Additives for the Stabilization of DoubleStranded DNA in UV-MALDI MS. J. Am. Soc. Mass Spectrom. 2002, 13, 1129-1137.

11. Zhu, Y. F.; Chung, C. N.; Taranenko, N. I.; Allman, S. L.; Martin, S. A.; Haff, L.; Chen, C. H. The Study of 2,3,4-Trihydroxyacetophenone and 2,4,6-Trihydroxyacetophenone as Matrices for DNA Detection in MatrixAssisted Laser Desorption/Ionization Time-of-Flight Mass Spectrometry. Rapid Commun. Mass Spectrom. 1996, 10, 383-388.

12. Song, F. H. Quinaldic Acid as a New Matrix for Matrix-Assisted Laser Desorption/ Ionization of Nucleic Acids. Rapid Commun. Mass Spectrom. 2003, 17, 1802-1807.

13. Zhou, L. H.; Deng, H. M.; Deng, Q. Y.; Zhao, S. K. A Mixed Matrix of 3-Hydroxypicolinic Acid and Pyrazinecarboxylic Acid for MatrixAssisted Laser Desorption/Ionization Time-of-Flight Mass Spectrometry of Oligodeoxynucleotides. Rapid Commun. Mass Spectrom. 2004, 18, 787-794.

14. Distler, A. M.; Allison, J. Improved MALDI-MS Analysis of Oligonucleotides Through the Use of Fucose as a Matrix Additive. Anal. Chem. 2001, 73, 5000-5003.

15. Kim, Y.; Hurst, G. B.; Study of Matrix and Polymer Substrate in MALDI-TOF Mass Spectrometry of DNA. Microchem. J. 2001, 70, 219228.

16. Kokotos, G.; Tzougraki, C. Synthesis and Study of Substituted Coumarins. A Facile Preparation of D,Lo-Tyrosine. J. Heterocyclic Chem. 1986, 23, 87-91.

17. Tang, K.; Allman, S. L.; Jones, R. B.; Chen, C. H.; Araghi, S. Laser Mass Spectrometry of Polydeoxyribothymidylic Acid Mixtures. Rapid Commun. Mass Spectrom. 1993, 7, 63-66. 